\title{
PROMOCIÓN DEL CAMBIO DE ESTILOS DE APRENDIZAJE Y MOTIVACIONES EN ESTUDIANTES DE EDUCACIÓN SUPERIOR MEDIANTE ACTIVIDADES DE TRABAJO COLABORATIVO EN BLENDED LEARNING
}

\author{
(PROMOTING CHANGES IN LEARNING STYLES AND MOTIVATIONS ON HIGHER \\ EDUCATION STUDENTS THROUGH COLLABORATIVE ACTIVITIES IN BLENDED \\ LEARNING)
}

Néstor Fernández Sánchez

Universidad Nacional Autónoma de México (México)

\section{RESUMEN}

Se realizó un estudio con 80 estudiantes mexicanos de educación superior para propiciar el cambio de sus motivaciones y estrategias de aprendizaje por medio de actividades orientadas al aprendizaje autogestionado y el trabajo colaborativo en la modalidad BLearning. Se aplicó el instrumento MSLQ en un diseño pretest - postest y se analizaron los datos de manera estadística. Los resultados indican que es posible modificar la Ansiedad, la Motivación extrínseca y la Búsqueda de ayuda, de acuerdo a las ideas de Donolo y colaboradores (2004). Se analiza la pertinencia de la consideración a los estilos de aprendizaje y la motivación en los procesos enseñanza - aprendizaje, en la modalidad a distancia, en BLearning.

Palabras clave: motivación, estilos de aprendizaje, blended learning.

\begin{abstract}
This article reports the findings of a study of 80 students of higher education in Mexico to promote changes in their motivation and learning strategies through self-managed learning oriented activities and collaborative work on b-learning. The MSLQ instrument was applied pre- and post-treatment. Statistical data were analyzed. The results indicated that anxiety, extrinsic motivation and search for help can be modified, according to the ideas of Donolo et al. (2004). We analyze the relevance of the consideration of learning styles and motivation for teaching and distance learning in b-learning.
\end{abstract}

Keywords: Motivation, learning styles, blended learning. 
Con el advenimiento de las Tecnologías de la Información y la Comunicación, la Educación a Distancia (EaD) ha venido a fortalecer su función en los procesos de formación y actualización profesional. Como en la modalidad presencial o semi presencial (Blended Learning o BLearning) (Reay, 2001), se requiere de una planeación cuidadosa para favorecer el logro de los objetivos de aprendizaje como consecuencia dela realización de las actividades programadas en los actos académicos. De igual manera, es indispensable orientar el proceso de enseñanza centrando la atención en el educando y, por supuesto, en el logro de su aprendizaje significativo (Gallego y Martínez, 2003). Lo anterior es una respuesta a las recomendaciones que hacen aproximaciones teóricas de la Psicología del aprendizaje, modelos pedagógicos y principios del Diseño Instruccional por rescatar la consideración hacia los estilos de aprendizaje del destinatario como elementos promotores de la construcción o apropiación del conocimiento. En la educación superior, la actualización profesional, la capacitación o en la educación continua es necesario ser puntual en estos temas toda vez que en estos contextos se trabaja con adultos. De manera específica, en la EaD o en su modalidad semi presencial (BLearning), se espera que el éxito de los actos académicos se vincule con los estilos de aprendizaje y la motivación del estudiante para la generación autónoma del conocimiento, actitudes y habilidades. (Alonso, Gallego y Honey, 1994; Alonso y Gallego, 2008). Algunas de las diversas formas de identificar los estilos de aprendizaje y las motivaciones han mostrado que éstos elementos no necesariamente forman parte del bagaje de los estudiantes (de manera evidente), no se aplican diferencialmente para contenidos que así lo requieren o no se orientan adecuadamente para el logro de los objetivos educacionales previstos en los programas educativos; lo anterior podría propiciar el fracaso, el abandono o el desvío de los avances del aprendizaje en los actos académicos en los que el estudiante depende del profesor o éste no se encuentra en plena disposición para orientar, atender las dudas o realimentar a los estudiantes.

Tomando en cuenta un estudio previo relacionado con los estilos de aprendizaje en los actos académicos, bajo la modalidad a distancia, en educación continua (Fernández, 2007), atrae ahora la idea por favorecer en el estudiante adulto la reconstrucción o reorientación de sus estilos de aprendizaje y sus motivaciones en actos académicos a distancia (Jacobson y Harris, 2008). Por ello, surgió la inquietud por desarrollar un estudio en el que se promoviera intencionalmente el ejercicio de estrategias de aprendizaje en actos académicos en la modalidad BLearning. De forma colateral surgió la preocupación acerca de si dichos elementos merecerían mayor atención en grupos de diferente edad. En este documento se reportan los avances de la intervención para promover las motivaciones en los estudiantes de una carrera universitaria, en una asignatura orientada a la comprensión y análisis del 
Desarrollo Humano. Es por lo anterior que se plantearon las siguientes preguntas de investigación:

¿Es posible modificar los estilos de aprendizaje y las motivaciones que influyen en el aprendizaje en estudiantes de educación superior?

¿Existen diferencias de estilos de aprendizaje entre mujeres y varones?

En su caso, ċen qué elementos motivacionales hay que incidir para favorecer el aprendizaje?

\section{EDUCACIÓN A DISTANCIA Y BLENDED LEARNING}

La educación a distancia puede ser abordada como objeto de estudio desde diferentes perspectivas. Existen diversos criterios para definirla: separación de los maestros y estudiantes, al menos en la mayor parte del proceso; el uso de comunicación en ambos sentidos entre estudiantes e instructores y el uso de los medios tecnológicos educacionales para unir a maestros y estudiantes (Álvarez, 1988). En los registros del Thesaurus de la Education Resources Information Center (ERIC) se asevera que es la educación desarrollada con los medios de comunicación (correspondencia, radio, televisión y otros), en salón de clase o no, con breves contactos cara a cara entre el estudiante y el profesor. También refiere que se trata del proceso para impartir u obtener conocimientos, actitudes, habilidades o valores sociales con apoyos que se ofrecen a distancia. Cabe señalar que desde 1966 la organización ERIC continúa agregando acepciones relacionadas con el tema en su Thesaurus, como Comunicación satelital, Comunicación mediada por computadora, Educación por radio, Televisión educativa, Programas externos de grado, Estudio en casa, Televisión interactiva, Programas externos, Telecursos, Online Courses, Web Based Instruction, Virtual Universities y Virtual Classrooms entre otros (ERIC, 2007). Un breve análisis histórico, conceptual y funcional sobre el tema se puede revisar en los trabajos de Álvarez (op. cit.) y Fernández (op. cit.). El tema que ocupa en el presente documento es la modalidad semi presencial, Blended Learning, $B$ Learning o BLearning en la que pueden incluirse diversas combinaciones del proceso enseñanza aprendizaje; desde una experiencia con amplia relación "cara a cara", acompañada de algunas relaciones "a distancia" (o viceversa) hasta la aplicación de diversos métodos de enseñanza en los que se hace uso de las Tecnologías de la Información y la Comunicación (Osguthorpe y Graham, 2003; ERIC, 2007). En el caso de la experiencia que aquí se expresa, se considera al BLearning como la oportunidad que permite al alumno disfrutar simultáneamente de las ventajas de una 
clase presencial, de una tutoría personalizada constante en línea y de una evaluación permanente de los avances de su aprendizaje, donde el docente desempeña el papel de orientador y supervisor del proceso de aprendizaje (Jerónimo, 2003; Cal, y Verdugo, 2009); así mismo, se asume la ventaja de la flexibilidad espacio-temporal y la abierta accesibilidad a los materiales (Sosa, Cortés, García, Sánchez y cols., 2005). Retomando a Osguthorpe y Graham (op. cit.), nos referimos a la instrucción que combina la relación cara a cara entre estudiante - docente y la instrucción mediada por los servicios de las Tecnologías de la Información y la Comunicación.

\section{LA EDUCACIÓN A DISTANCIA EN MÉXICO Y EL USO DE LAS TIC}

Esta modalidad adquiere una mayor participación en el contexto de la educación mundial, prueba de ello se encuentra en la Declaración Mundial sobre la educación superior en el Siglo XXI (UNESCO, 1998). Una muestra importante del interés por este tema se puede rescatar de las páginas de PubliCUED, de la Cátedra UNESCO para América Latina (www.uned.es). Atender la educación a distancia es parte de los desafíos que las Instituciones de Educación Superior deben asumir como parte de los retos del Siglo XXI, según ha expuesto de manera frecuente Tünnermann (1999, 2003, 2007, 2010; UNISON,1999).

En México, diversas instituciones han encontrado a la Educación a Distancia como una fuerte opción para compartir con más personas el conocimiento que se genera en sus aulas y centros de investigación (Vega, 2005; Torres, 2006). Esta modalidad educativa se ha impulsado por la Asociación Nacional de Universidades e Instituciones de Educación Superior (ANUIES, 2000), la Universidad Nacional Autónoma de México (www.cuaed.unam.mx), el Instituto Politécnico Nacional (www.polivirtual.ipn.mx) y diversas organizaciones educativas que comienzan a posicionarse en este tema, como el Espacio Común de Educación Superior a Distancia (ECOESAD, www.ecoesad.org.mx), la Asociación Mexicana de Educación a Distancia AMECyD (www.amecyd.uaemex.mx) y la UDGVIRTUAL (www.udgvirtual.udg. $\mathrm{mx}$ ), entre otras. Detalles de la evolución de este tema en México, se expresan en los libros Educación, estandarización y tecnología (Pastor, 2006), La educación a distancia en México: narrativa de una historia silenciosa (Bosco y Barrón, 2009) y La Educación Superior a Distancia en América Latina y el Caribe (Amador, 2010).

Aunado a lo anterior, es necesario considerar el número creciente de usuarios de Internet y que aprovechan la Tecnología para atender asuntos de educación. La Asociación Mexicana de Internet expone que la mayoría de los usuarios de Internet 
son adultos y estudiantes. Se calcula que en el año 2010 hubo cerca de 30 millones de usuarios (AMIPCI, 2009).

\section{ESTILOS DE APRENDIZAJE}

Este tema tiene un especial interés toda vez que se atribuye al estudiante una gran parte de las causas de la deserción o, por el contrario, la eficiencia en los programas académicos. No obstante que la Eficiencia Terminal (y contraparte, la deserción) es un indicador que permite a las instituciones educativas reformar su planeación educativa, es difícil que alguna de ellas reporte públicamente sus datos; más difícil es encontrar datos derivados de la Educación Continua o de ésta en sus diversas modalidades. En términos generales, se estima que la deserción escolar alcanza un promedio de 50\%, según reportan la OCDE, UNESCO y ANUIES (Fernández, 2005). Al parecer, la deserción se incrementa en modalidades diferentes a lo presencial (Vásquez, 2003; Vásquez y Rodríguez, 2007; Hernández, Rama, Jiminián y Cruz, 2009; Zubieta, Cervantes y Rojas, 2009).

Al respecto, Cervantes (2004) exploró algunas de las posibles causas de la deserción de los profesores - alumnos que formaron como formadores de educación en línea, en el Instituto Latinoamericano de Comunicación Educativa. El análisis cualitativo que realizó acerca de las opiniones de los desertores dio como resultado una clasificación de posibles variables de influencia: Administrativas, actitudinales, procedimentales y a causa de las estrategias de aprendizaje. De manera específica, comenta que los participantes... (a) se sienten abandonados cuando no obtiene respuesta inmediata a sus correos, (b) no reciben atención personalizada (c), se molestan cuando sus trabajos no son revisados con profesionalismo, (d) no admiten el punto de vista de otros, (e) carecen de habilidades para la lectura de comprensión y (f) no asumen el papel de alumnos y rechazan las observaciones de los tutores. Similares observaciones hace Padula (2005) y agrega que la participación del conductor o facilitador del aprendizaje es una variable de influencia en la posible deserción en la EaD. Además, se ha observado que el rendimiento escolar influye por el tipo de hábitos de estudio o los estilos de aprendizaje con los que cuentan los alumnos; situación en la que coincide Esteban y Zapata, (2008).

El tema de los Estilos de aprendizaje, extendido en su estudio desde la década de los 70's, Marton y Saljo, (1976) han revisado diversas expectativas y explicaciones. Afirmaciones de corte pedagógico afirman que dichos estilos se refieren a las diversas formas en que el estudiante puede apropiarse del conocimiento (Weinstein y Mayer, 1986). Igualmente, poseemos diferentes estilos de aprendizaje y éstos son 
una importante cuestión a tomar en cuenta en el diseño, ejecución y control del proceso de aprendizaje (Diaz - Barriga y Hernández, 2001) ya que los profesores pueden ayudar a sus estudiantes concibiendo una instrucción que responda a sus necesidades y a los diferentes estilos, orientándoles para que mejoren sus estrategias de aprendizaje. Llámese entonces estilos de aprendizaje a los hábitos para procesar la información, relacionados con la percepción, pensamiento, memoria y la solución de problemas (ERIC, 2007).

Los estilos de aprendizaje, de manera específica su tipología, fueron descritos por David Kolb en su modelo de aprendizaje experiencial. De acuerdo con Kolb (1983), el ciclo de aprendizaje experiencial tiene cuatro fases: la experiencia concreta, la observación reflexiva, la conceptualización abstracta y la experimentación activa. Cada una implica un modo diferente de experiencia de la realidad. Con base a estas ideas, Honey y Mumford, (1983) retoman la idea de un modelo de aprendizaje experiencial en cuatro fases denominadas: la experiencia, el regreso sobre la experiencia, la formulación de conclusiones y la planificación. Estos autores afirman que la mayoría de las personas, a través de los logros y los fracasos de sus conductas en sus tentativas de aprender, desarrollan preferencias que los hacen apropiarse particularmente de ciertas fases del proceso y, con ello, de estilos diferentes de aprendizaje. Una de las aportaciones colaterales a sus trabajos fue el desarrollo de un instrumento exploratorio de los estilos de aprendizaje.

Hoy en día, la literatura especializada en educación aplica el concepto de estilos de aprendizaje para referirse a las reglas, principios, y procedimientos que suelen facilitar el aprendizaje y que, con frecuencia se aplica a una variedad de tareas de estudio.

La mayoría de los documentos recientes que versan sobre el tema retoman el punto de vista de Mainemelis y colaboradores quienes, incluso, han aportado formas de evaluación para identificar los estilos que el estudiante ocupa para el aprendizaje. Las aportaciones de éstos estudiosos, y en especial de David Kolb, sugieren que el estudiante puede asumir con sus estrategias algunas de las siguientes categorías: Alumno activo, reflexivo, teórico o pragmático (Kolb, op. cit.; Camarero, Martín del B, y Herrero, 2000; Mainemeli, Boyatizus y Kolb, 2002).

Retomando las aportaciones de Donolo y cols., los estudiantes pueden asumir estrategias cognitivas, metacognitivas y de regulación de recursos (Rinaudo, Chiecher, y Donolo, 2003; Donolo, Chiecher, y Rinaudo, 2004 y 2008): 
- Metas de orientación intrínseca (MI). Refiere al grado en que los estudiantes realizan las tareas y acciones por el interés que les genera la actividad misma.

- Metas de orientación extrínseca (ME). Evalúa el grado en que los sujetos realizan una determinada acción 'para' satisfacer otros motivos que no están relacionados con la actividad en sí misma, sino en la consecución de otras metas.

- Valoración de la tarea (VT). Alude a la evaluación que hace el estudiante de cuán interesantes, importantes y útiles son las actividades o materiales del curso.

- Creencias de control del aprendizaje (CC). Evalúa las creencias de los estudiantes acerca del grado de control que tienen sobre su propio aprendizaje.

- Creencias de autoeficacia (CA). Refiere a las percepciones de los estudiantes sobre su capacidad para desempeñar las tareas requeridas en el curso.

- Ansiedad (AN). Alude al grado de ansiedad de los estudiantes frente al aprendizaje.

- Las estrategias de repaso (EP) son los procedimientos más simples e incluyen operaciones básicas que favorecen el recuerdo de la información mediante repetición o recitación.

- Las estrategias de elaboración (EL) constituyen un nivel intermedio entre las estrategias de repaso y de organización y permiten una transformación de la información así como el establecimiento de conexiones entre los conocimientos del sujeto y los proporcionados por el nuevo material.

- Las estrategias deorganización(EO) conducirían a procesamientos más profundos de los materiales de estudio, permitiendo construir conexiones internas entre las piezas de información ofrecidas en el material de aprendizaje.

- El pensamiento crítico (PC) es considerado también como una estrategia cognitiva, que refiere al intento de los estudiantes de pensar de un modo más profundo, reflexivo y crítico sobre el material de estudio.

- La Autorregulación cognitiva (AM) sugiere que hay tres procesos generales: el planeamiento, el control y la regulación. Planear las actividades contribuye para activar aspectos relevantes del conocimiento previo, que permiten organizar y comprender más fácilmente el material. Controlar las actividades implica evaluar la atención y cuestionarse durante la lectura, en tanto que la regulación de las actividades refiere al continuo ajuste de las acciones cognitivas que se realizan en función del control previo. 
- El manejo del tiempo (MT) implica programar y planear los momentos de estudio, en tanto que el manejo del ambiente refiere a la determinación por parte del estudiante acerca de su lugar de trabajo. Idealmente, el ambiente de estudio debe ser tranquilo, ordenado y relativamente libre de distractores.

- La regulación del esfuerzo (ES) alude a la habilidad del estudiante para persistir en las tareas a pesar de las distracciones o falta de interés; tal habilidad es de importancia para el éxito académico en la medida que implica compromiso con las actividades y tareas propuestas.

- El aprendizaje con pares (AP) alude a la disposición de los estudiantes para compartir su aprendizaje y trabajar de manera colaborativa.

- La búsqueda de ayuda (BA) refiere a la disposición para recurrir a compañeros o al docente para plantear las dificultades en la realización de actividades orientadas al aprendizaje.

Diversos enfoques e instrumentos se han procurado para explorar o medir los estilos de aprendizaje, entre ellos: el Test de Emparejamiento de Figuras Familiares (Matching Familiar Figures Test), de Kagan (1966); Cuestionario de Estilos de Aprendizaje de Estudiantes (Student Learning Styles Questionnaire), de Grasha y Riechmann (1974); Inventario de Estilos de Aprendizaje (Learning Style Inventory), de Kolb (1976); Perfil de Estilos de Aprendizaje (Learning Style Profile), de Keefe (1979); Cuestionario Índice de Estilo de Aprendizaje (Index of Learning Styles), de Felder y Silverman (1988); Cuestionario de Estilos de Aprendizaje (Learning Styles Questionnaire), de Honey y Mumford (1988) y los Inventarios de Estilos de Aprendizaje- Version III (Learning Styles Inventory--Version III-Elementary Class Set), de Renzulli, Smith, y Rizza (2002) citados por García (2008).

Con base a una experiencia previa Fernández (2007) en la exploración de los estilos de aprendizaje con el instrumento de Honey y Alonso (Alonso, Gallego y Honey, 1994), se instauraron actividades de aprendizaje para estudiantes de educación superior, bajo la modalidad de BLearning, para atender la compresión y análisis de contenidos relacionados con el Desarrollo Humano (Psicología del desarrollo o Psicología evolutiva) y aspectos orientados a la educación. Durante tres meses se trabajó en sesiones presenciales de análisis de las lecturas y actividades de aprendizaje que se solicitaron en Internet, con apoyo de la plataforma Moodle. Para atender los contenidos propios del programa escolar, se propició el trabajo colaborativo y el estudio independiente a fin de coadyuvar con el estudiante en la ampliación o fortalecimiento de estrategias de aprendizaje. 
Con base a lo anterior, se estableció la siguiente hipótesis de trabajo:

Los estilos de aprendizaje y motivaciones del estudiante de educación superior, en actos académicos desarrollados bajo BLearning, se modifican cuando las actividades de aprendizaje se orientan hacia el trabajo colaborativo y el aprendizaje autogestionado.

\section{Método}

\section{Muestra}

Se contó con la colaboración de 47 estudiantes mexicanos, 39 mujeres y 8 hombres. Los criterios de inclusión fueron: aceptar voluntariamente su participación, ser estudiantes de los programas educativos bajo la coordinación del mismo profesor y haber concluido satisfactoriamente su desempeño en las actividades y tareas asignadas en el programa. La población original fue de 50 estudiantes, tres de ellos no cumplieron alguno de los criterios mencionados.

\section{Instrumentos}

Cuestionario de Motivación y Estrategias para el Aprendizaje (MSLQ). El instrumento está conformado por 81 ítems que se responden sobre la base de una escala Likert de 7 puntos. Se adaptó el cuestionario para su aplicación y calificación de manera automatizada por medio de formularios en un sitio Web. Incluidos en los formularios se registraron las variables género, edad, nivel de estudios y otros datos para una investigación paralela. Aspectos metodológicos del MSLQ han sido detallados por Pintrich (1991), quien elaboró con sus seguidores el instrumento Motivated Strategies for Learning Questionnaire o MSLQ (Pintrich, Smith, García y McKeachie, 1991; Pintrich y García, 1993). Este instrumento, que cuenta con validez comprobada (Antino, 2005; Cardozo, 2008; Paolini, Chiecher y Donolo, 2008) ha sido aplicado para identificar las diferencias de estilos de aprendizaje, tanto en la modalidad presencial como en la EaD (Pintrich y García, 1993; Suárez y Anaya 2004; Richardson, 2007; Hergul, 2004; Torres y Eberle, 2010; Miltiadou y Wilhelmina, 2003; Bassili, 2008; Yukselturk y Bulut, 2009). Para la población hispanoparlante se cuenta con la versión validada de este instrumento desarrollada en España (Roces, Tourón y González, 1998) y una versión adaptada a Latinoamérica (Donolo, Rinaudo, Chiecher y Paoloni, 2008). 


\section{Variables dependientes}

Motivación y estrategias de aprendizaje. Cuantificación de las características de cada una de las escalas del instrumento MSLQ:

- Metas de orientación intrínseca (MI). Refiere al grado en que los estudiantes realizan las tareas y acciones por el interés que les genera la actividad misma.

- Metas de orientación extrínseca (ME). Evalúa el grado en que los sujetos realizan una determinada acción 'para' satisfacer otros motivos que no están relacionados con la actividad en sí misma, sino en la consecución de otras metas.

- Valoración de la tarea (VT). Alude a la evaluación que hace el estudiante de cuán interesantes, importantes y útiles son las actividades o materiales del curso.

- Creencias de control del aprendizaje (CC). Evalúa las creencias de los estudiantes acerca del grado de control que tienen sobre su propio aprendizaje.

- Creencias de autoeficacia (CA). Refiere a las percepciones de los estudiantes sobre su capacidad para desempeñar las tareas requeridas en el curso.

- Ansiedad (AN). Alude al grado de ansiedad de los estudiantes frente al aprendizaje.

\section{Variables Independientes}

- Recomendaciones para el mejoramiento de la aplicación de estilos y estrategias de aprendizaje.

- Actividades de aprendizaje orientadas hacia el estudio independiente y hacia el trabajo colaborativo.

- Género. Femenino y masculino. Rasgo biológico inherente a las personas de acuerdo al rol sexual: mujeres u hombres.

\section{Procedimiento}

Fase 1.- Levantamiento de datos. Una vez iniciado el curso del programa educativo, se exhortó a los participantes a resolver el instrumento.

Fase 2.- Intervención 
En la primera sesión se instruyó a los participantes en la forma de trabajo y forma de evaluación por medio de rúbricas. Se distribuyeron claves de acceso para ingresar a la plataforma Moodle y se hizo hincapié en la resolución del cuestionario. En la segunda semana se reiteraron los puntos antes mencionados. A la conclusión del cuestionario, dependiendo de los puntajes obtenidos (cuando éstos eran inferiores a la media aritmética esperada) el sistema aportó al estudiante una serie de recomendaciones para fortalecer sus estilos de aprendizaje.

Durante 16 semanas se trabajó con la plataforma para distribuir materiales de apoyo y las actividades de aprendizaje. La mayoría de éstas se orientaron hacia el estudio independiente y al trabajo colaborativo, en equipos de 5 a 6 participantes. En sesiones presenciales, se realizó el análisis de los contenidos revisados y la utilidad de estos. Los resultados del trabajo individual y colaborativo se fueron evaluando de manera formativa conforme se realizaban las entregas programadas, asignando la calificación de acuerdo a los criterios expuestos en las rúbricas. De esta forma, los estudiantes obtuvieron realimentación inmediata, teniendo la oportunidad de mejorar el resultado de su esfuerzo. Con base a lo anterior, no se realizó evaluación sumativa.

Fase 3.- Una vez concluido el curso y confirmadas las calificaciones obtenidas, se invitó de nuevo a resolver el cuestionario.

\section{Resultados}

El análisis de los datos del instrumento arrojó una Alfa de Cronbach de o.8808, lo que indica una adecuada consistencia interna del MSLQ en la población explorada.

La comparación de medias de calificaciones para cada una de las escalas del MSLQ, antes y después de la intervención, reflejan una aparente diferencia en la Ansiedad (AN), Pensamiento crítico (PE) y Búsqueda de ayuda (BA). No obstante, el nivel de significancia de estas fue de $0.119,0.362$ y 0.239 respectivamente (véase Gráfico 1). 


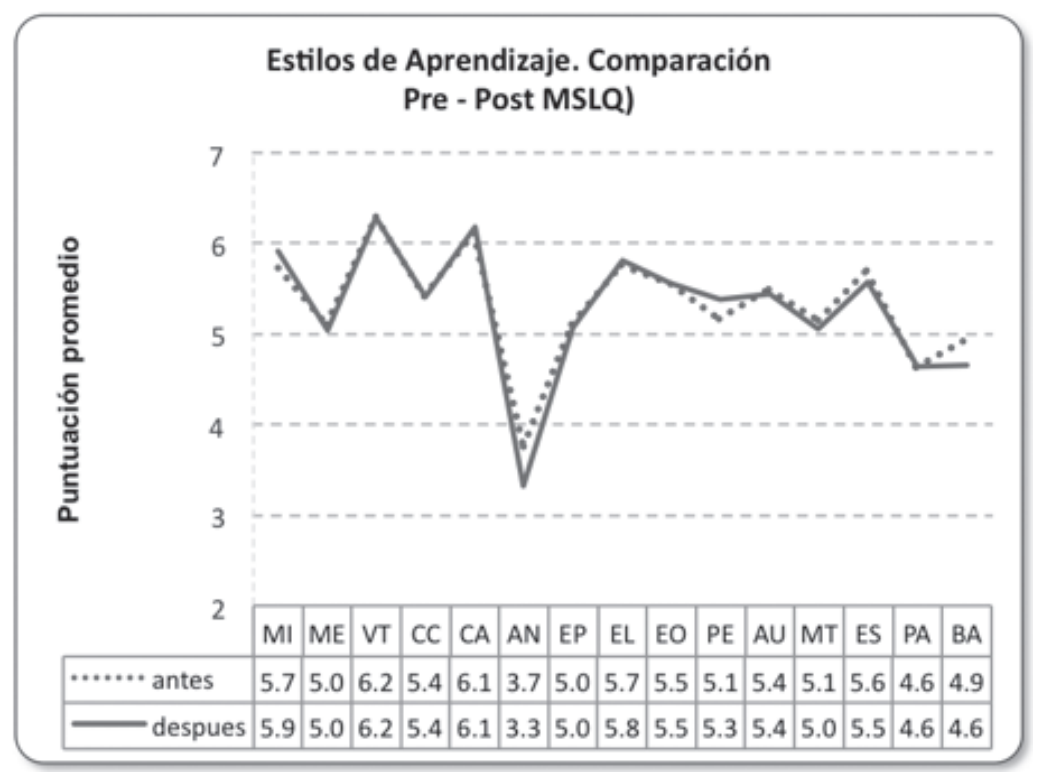

Gráfico 1. Comparación de promedios pre - post, todos los participantes

Los primeros resultados no reflejan diferencias significativas, esto puede deberse a un aspecto andragógico y de género, identificado previamente (Fernández, 2007). Tomando en consideración la posibilidad de encontrar diferencias entre géneros, se realizó un análisis estadístico para comparar entre mujeres y varones.

La comparación de medias de calificaciones, para cada uno de los estilos de aprendizaje en el grupos de mujeres, reporta diferencias significativas de 0.013, con $\mathrm{NS}=0.00$, en la escala de Ansiedad (AN), siendo la media inicial de 3.9 y de $3.4 \mathrm{al}$ término del curso en la prueba estadística T- Test. (Véase Gráfico 2).

Para el caso de la comparación de las medias en los varones, se muestran diferencias significativas en las Metas de orientación extrínseca (ME) y Búsqueda de ayuda $(\mathrm{BA})$, con valores $0.040(\mathrm{NS}=0.00)$ y $0.014(\mathrm{NS}=0.022)$ respectivamente en la prueba estadística T- Test. Los valores de las medias aritméticas previas y posteriores a la intervención fueron de 4.7 y de 4.3 en las Metas de orientación extrínseca (ME) y de 4.8/ 4.1 en la Búsqueda de ayuda. (Véase Gráfico 3). 
Promoción del CAmbio de Estilos de AprendizAje y Motivaciones en Estudiantes...

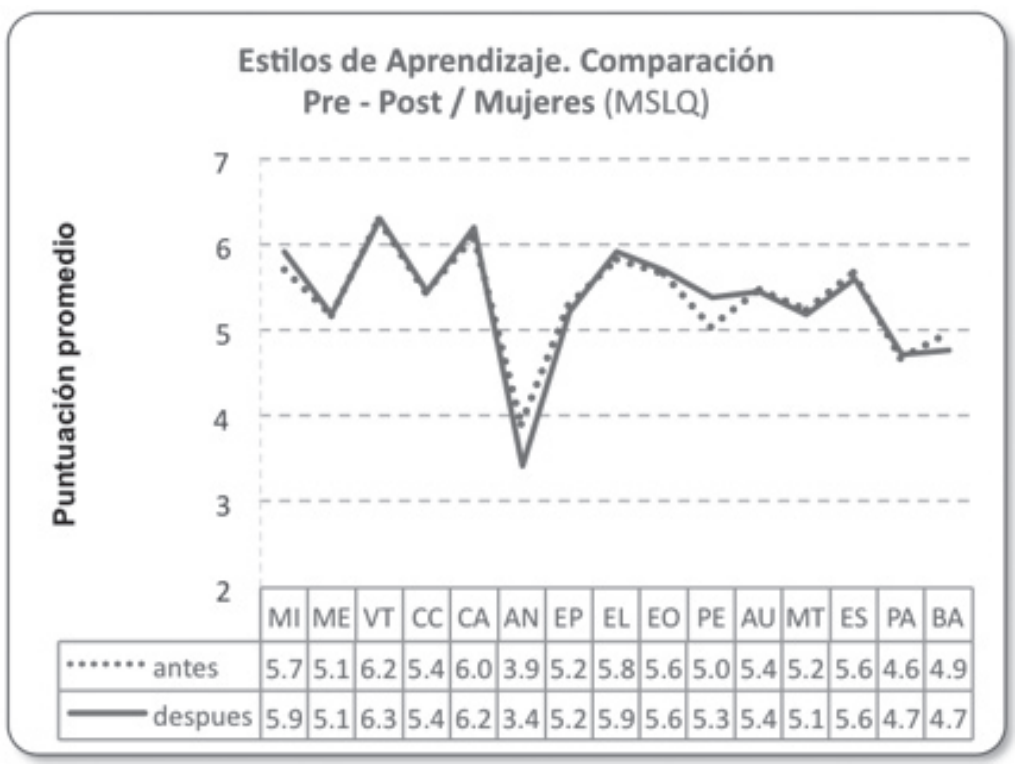

Gráfico 2. Comparación de promedios pre - post en mujeres

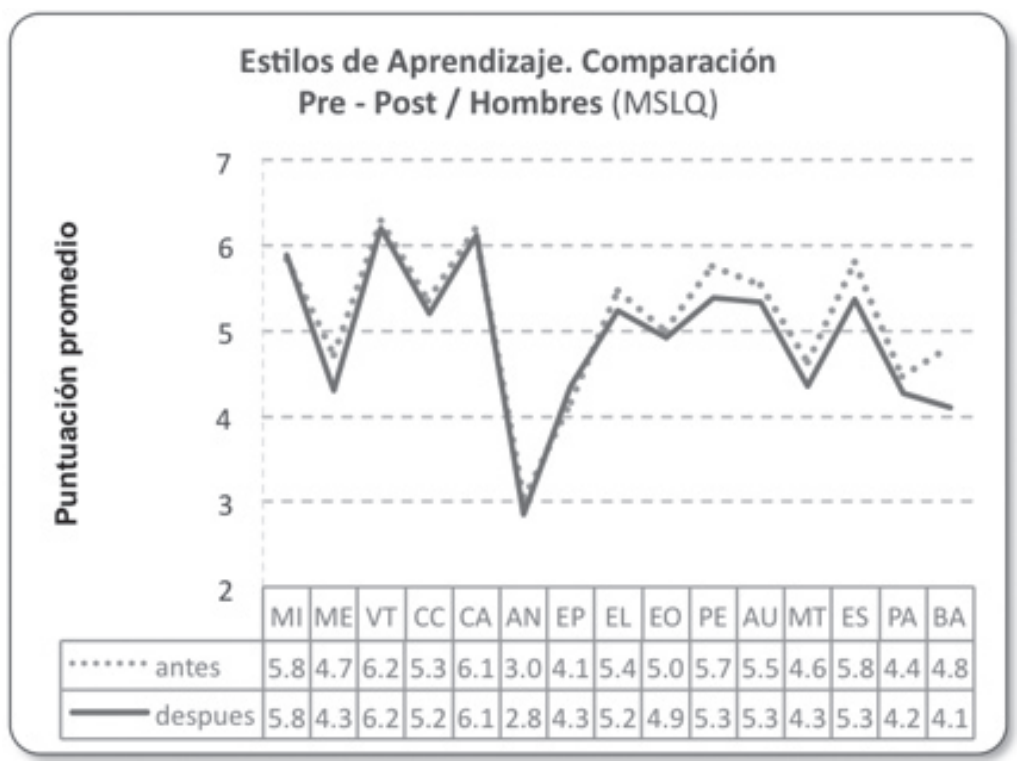

Gráfico 3. Comparación de promedios pre - post en varones 


\section{ANÁLISIS Y CONCLUSIÓN}

Como se puede apreciar en los datos y los gráficos, existen diferencias significativas en los promedios de la escala de Ansiedad, sólo en las mujeres; la media aritmética de la escala disminuyó después de la intervención. De forma similar, este valor disminuyó en la escala Metas de orientación extrínseca en el caso de los varones. Caso similar se muestra en la escala de Búsqueda de ayuda, toda vez que las medias aritméticas disminuyeron en ambos grupos una vez concluida la intervención.

Lo anterior permite confirmar parcialmente las hipótesis, ya que algunos de los estilos de aprendizaje y motivaciones del estudiante de educación superior, en actos académicos desarrollados bajo BLearning, se modifican cuando las actividades de aprendizaje se orientan hacia el trabajo colaborativo y el aprendizaje autogestionado. En este caso, se disminuyeron los valores de las escalas Ansiedad, en mujeres, y Metas de orientación extrínseca en los varones. En ambos casos, fue Búsqueda de ayuda la que disminuyó.

Con base a lo anterior, es posible que los estilos de aprendizaje y Estrategias cognitivas del estudiante de educación superior sean diferentes entre mujeres y hombres.

Los resultados sugieren que la Ansiedad, derivada de la las formas de evaluación del aprendizaje, disminuyó en las mujeres. Esta situación ideal, la no ansiedad ante la evaluación, es una expectativa conveniente para el estudiante toda vez que la disposición para ser evaluado podría formar parte de su seguridad, aunado a la mejoría de sus motivaciones intrínseca y extrínseca. Al respecto, existen propuestas para apoyar al estudiante a fin de disminuir dicha ansiedad (Alvarez, y cols., 2008; Bausela, 2005) y una serie de consideraciones relacionadas con la motivación del estudiante (Paoloni, 2008).

Con relación a la Motivación extrínseca de los varones, es interesante identificar el grado en que estos realizan las actividades de aprendizaje para satisfacer otros motivos que no están relacionados con la actividad en sí. Definitivamente, es deseable que dicha motivación disminuya para que pase a ser, de un interés controlado por el entorno o por la competencia social, por aquella que satisfaga al estudiante por la propia razón de aprender, tema sugerido por las aportaciones del enfoque constructivista (Diaz - Barriga y Hernández, 2001). Para el caso de la EaD -en este caso, BLearning-, la motivación extrínseca pasa a segundo plano cuando el objetivo es que el estudiante se convierta en agente autogestivo del aprendizaje. 
Respecto a la disminución de la búsqueda de ayuda, como en el caso de la motivación extrínseca, se espera que el estudiante promueva su aprendizaje de forma independiente, sin respaldarse necesariamente en sus pares o el docente. De esta forma, se promueve el aprender a aprender y se perfilará hacia la construcción del conocimiento de manera propiocetiva.

En términos generales, se espera que este tipo de experiencias apoyen el proceso educativo en estudiantes de nivel superior, en los actos académicos desarrollados en la modalidad a distancia, específicamente en la forma de trabajo semi presencial o BLearning. Como apunta Moreno (1997), el aprendizaje autogestivo no es un objetivo a priori de la $\mathrm{EaD}$ (en este caso BLearning), más bien hay que procurar entornos de apoyos tecnológicos y académicos para desarrollar ambientes propicios para el aprendizaje autogestivo.

Es necesario mencionar que, debido a la imposibilidad de crear un escenario tipo laboratorio para mantener posibles variables extrañas que intervengan en los resultados, es probable que otras variables, además de las consideradas en la intervención aquí expuesta, pudieran tener relación con los cambios aquí aportados, tales como la edad de los sujetos, o estado de maduración cognitiva, o la forma de trabajo, o estilos de enseñanza del profesor que dirigió las asignaturas paralelas. Es decir, las variables extrañas (Campbell y Stanley, 1973). Por este motivo, se recomienda, en estudios como éste, atender con mayor precisión principios metodológicos para fundamentar posibles generalizaciones.

\section{REFERENCIAS BIBLIOGRÁFICAS}

Alonso, G. C.; Gallego, G. D.; Honey, P. (1994). Los Estilos de Aprendizaje. Procedimientos de diagnóstico y Mejora (6 ed.). Bilbao, España: Ediciones Mensajero.

Alonso, C. M.; Gallego, G. J. D. (2008). CHAEA. Estilos de aprendizaje [en línea] Disponible en: www. estilosdeaprendizaje.es (consulta 2009, 5 de marzo).

Alvarez G. D. (1988). Educación a Distancia. ¿Para qué y cómo? (R. T. Cuba, Ed.) Cuba: Editorial InfoMed.

Alvarez, H. J.; Carrión, M. J. J.; Casanova, A. P. F.; Rubio H. R.; Miras, M. F.;
Salvador, G. M. y cols. (2008). Programa autoaplicado para el control de la ansiedad antelos exámenes. Universidad de Almería. [en línea] Disponible en: http://www.ual.es/Universidad/ GabPrensa/controlexamenes/index.htm (consulta 2010, 10 de febrero).

Amador, B. R. (2010). La Educación Superior a Distancia en México. Realidades y tendencias. En: Lupion, T.; Rama, C. (Edits.). La Educación Superior a Distancia en América Latina y el Caribe Brasil: UNISUL (129 - 144).

AMIPCI. (2009). Hábitos de los Usuarios de Internet en México. México: Asociación 
Mexicana de Internet. [en línea]. Disponible en: http://estudios.amipci. org. $\mathrm{mx}: 8080 / \mathrm{mashboard} / \mathrm{main} . j \mathrm{sp}$ (consulta 2010, 17 de febrero).

ANUIES, (2000). Plan Maestro de Educación Superior Abierta y a Distancia. México: Asociación Nacional de Universidades e Instituciones de Educación Superior. Líneas estratégicas para su desarrollo.

Artino, A. R. (2005). Review of the Motivated Strategies for Learning Questionnaire. [en línea]. Disponible en: http://www.eric.ed.gov/ERICWebPortal/ contentdelivery/servlet/ERICServlet?acc

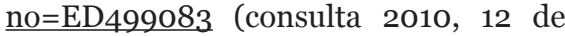
enero).

Bassili, J. N. (2008). Motivation and Cognitive Strategies in the Choice to Attend Lectures or Watch Them Online. Journal of Distance Education , 22 (3).

Bausela, H. E. (2005). Ansiedad ante los exámenes: evaluación e intervención psicopedagógica. Revista EDUCERE, 9 (31), (553-558). [en línea]. Disponible en: $\quad \underline{\text { http://redalyc.uaemex.mx/ }}$ pdf/356/35603117.pdf (consulta 2011, 15 de febrero).

Bosco, H. M. D.; Barrón, S. H. S. (2009). La educación a distancia en México: narrativa de una historia silenciosa. México: UNAM.

Cal, B. M. I.; Verdugo, M. V. (2009). Una experiencia b-learning en Econometría. Revista de Formación e Innovación Educativa Universitaria, 2 (4), (360366).

Camarero, S. F.; Martín del B., F.; Herrero, D. J. (2000). Estilos y estrategias de aprendizajeen estudiantes universitarios. Revista Psicothema, 12 (4), (615-622).

Campbell, D. T.; Stanley, J. C. (1973). Diseños experimentales y cuasiexperimentales en la investigación social. Buenos Aires: Amorrortu Editores.

Cardozo, A. (2008). Motivación, aprendizaje y rendimiento académico en estudiantes del primer año universitario. Laurus, 14
(28), (209-237). Universidad Pedagógica Experimental Libertador, Venezuela [en línea]. Disponible en: http://redalyc. uaemex.mx/pdf/761/76111716011.pdf (consulta 2011, 10 de febrero).

Cervantes, M. E. (2004). Deserción: Un análisis de la deserción en la modalidad de educación en línea de Red Escolar. Revista e-formadores, Instituto Latinoamericano de Comunicación educativa, 4, [en línea]. Disponible en: http://e-formadores.redescolar.ilce. edu.mx/revista/no7 05/ponencia.pdf (consulta 2005, 17 de enero).

Díaz-Barriga, A. F.; Hernández, R. G. (2001). Estrategias docentes para un aprendizaje significativo. Una interpretación Constructivista. México: McGraw Hill.

Donolo, D.; Chiecher, A.; Rinaudo, M. C. (2004). Estudiantes en entornos tradicionales y a distancia. Perfiles motivacionales y percepciones del contexto. Revista de Educación a Distancia,10, (1-14).

Donolo, D.; Rinaudo, M. C.; Chiecher, A.; Paoloni, P. V. R. (2008). MSLQe y MSLQvv. Propuestas para la medición de la motivación y el uso de estrategias de aprendizaje. Córdoba, Argentina: EFUNARC, Rio Cuarto ed.

ERIC (2007). Educational Resources Information Center. Thesaurus. [en línea]. Disponible en: www.eric.ed.gov (consulta 2010, 11 de noviembre).

Esteban, M.; Zapata, M. (2008). Estrategias de aprendizaje y eLearning. Un apunte para la fundamentación del diseño educativo en los entornos virtuales de aprendizaje. Consideraciones para la reflexión y el debate. Introducción al estudio de las estrategias y estilos de aprendizaje. Red Revista de Educación a Distancia, 19.

Fernández, S. N. (2005). Factores psicopedagógicos de influencia en la deserción de actos académicos de 
educación continua, por Internet. México: Tesis de maestría, Facultad de Psicología, Universidad Nacional Autónoma de México.

Fernández, S. N. (2007). Diferencias de Estilos de Aprendizaje entre jóvenes y adultos. Consideraciones andragógicas para la Educación Continua y a distancia, basadas en la investigación. III Congreso Internacional de AMECYD Redes de Cooperación. [en línea]. Disponible en: $\quad$ http://www.e-continua.com/ documentos/amecyd2007 estilos aprend.pdf Tijuana, B. C., México. ISBN 970-735-086-5. (consulta 2010, 15 de marzo).

Gallego-Rodríguez, A.; Martínez-Caro, E. (2003). Estilos de aprendizaje y elearning. Hacia un mayor rendimiento académico. RED. Revista de Educación a Distancia. [en línea]. Disponible en: http://redalyc.uaemex.mx/redalyc/src/ inicio/ArtPdfRed.jsp?iCve $=54700703$. (consulta 2010, 15 de enero).

García, G. J. L. (2008). Web de José Luís García Cue. [en línea]. Disponible en: http://www.jlgcue.es/ (consulta 2011, 17 de febrero).

Hergul, H. (2004). Relationship Between Student Characteristics and Academic Achievement in Distance Education and Application on Students of Anadolu University. Turkish Online Journal of Distance Education , 5 (2), (81-90).

Hernández, A.; Rama, C.; Jiminián, Y.; Cruz, M. (2009). Deserción en las instituciones de educación superior a distancia en América Latina y el Caribe. República Dominicana: Universidad Abierta para Adultos.

Honey, P.; Mumford, A. (1983). Using Your Learning Styles. Maidenhead. Reino Unido: Peter Honey Publications.

Jacobson, R. R.; Harris, M. S. (2008). Does the Type of Campus Influence SelfRegulated Learning as Measured by the Motivated Strategies for Learning
Questionnaire (MSLQ)? Education, 128, (412-431).

Jerónimo, M. J. A. (2003). Una experiencia de Formación docentes para la educación digital. RED. Revista de Educación a Distancia, 8.

Kolb, D. A. (1983). Experiential Learning: Experience as the Sourceof Learning and Development. New Jersey: PrenticeHall.

Mainemeli, C.; Boyatizus, R. E.; Kolb, D. A. (2002). Adaptive Flexibility. Testing Experiential Learning Theory of Development. Management Learning, 33 (1), (5-33).

Marton, F.; Saljo, R. (1976). On quelitative differencies on learning. 1 Outcome and process. Brit. J. Educ. Psychol. 46, (411).

Miltiadou, M.; Wilhelmina, C. S. (2003). Applying Social Cognitive Constructs of Motivation to Enhance Student Success in Online Distance Education. Educational Technology Review, 11 (1).

Moreno, C. M. (1997). El Desarrollo de Ambientes de Aprendizaje a Distancia. Revista Red Escolar. VI Encuentro Internacional de Educación a Distancia. Medios y modos de Aprender en la Educación a Distancia. Universidad de Guadalajara. [en línea] Disponible en: http://redescolar.ilce.edu.mx/ redescolar/biblioteca/articulos/pdf/ EL DESARROLLO DE AMBIENTES DE APRENDIZAJE.pdf (consulta 2009, 18 de mayo).

Osguthorpe, R. T.; Graham, C. R. (2003). Blended Learning Environments. Definitions and Directions. The Quarterly Blended learning systems. Review of Distance Education, 4 (3), (227-233).

Padula, P. J. E. (2005). Tutor y estudiante en educación a distancia. ¿̇Interacción motivadora o comunicación en crisis? Quaderns Digitals, 40. [en línea] Disponible en: http://www. lenguaweb.info/competencias-tic-del- 
profesorado/521-tutor-y-estudianteen-educacion-a-distancia-iinteraccionmotivadora-o-comunicacion-en-crisis (consulta 2010, 15 de mayo).

Paolini, P. V.; Chiecher, A.; Donolo, D. (2008). Evaluación Técnica del MSLQ para su aplicación en el contexto de la Universidad Nacional de Río Cuarto. Revista Contextos de Educación, VII (8), (135-145).

Paoloni, P. V. (2008). Conocimientos y habilidades metamotivacionales. Algunas implicancias para la motivación académica en la universidad. Contextos de Educación, VII (9), (328-338).

Pastor, A. P. (2006). Educación, estandarización y tecnología. México. Reseña por Corona, T. L. Revista Apertura, 6 (7), (106-107). [en línea] Disponible en: http://redalyc.uaemex. $\underline{\mathrm{mx} / \mathrm{pdf} / 688 / 68800610 . p d f}$ (consulta 2010, 10 de julio).

Pintrich, P.; Smith, D.; Garcia, T.; Mc Keachie, W. (1991). A Manual for the Use of the Motivated Strategies for Learning Questionnaire (MSLQ). Teaching and Learning.USA: National Center for Research to Improve Postsecondary. U. Michigan, Ed.

Pintrich, P.; García, T. (1993). Intraindividual differences in students' motivation and selfregulated learning. German Journal of Educational Psichology , 7 (3), (99107).

Reay, J. (2001). Blended Learning-a fusion for the future. Knowledge Management Review, 4 (6).

Richardson, J. T. (2007). Motives, attitudes and approaches to studying in distance education. Higher Education, 54 (3), (385-416).

Rinaudo, M. C.; Chiecher, A.; Donolo, D. (2003). Motivación y uso de estrategias en estudiantes universitarios. Anales de psicología , 19 (1), (107-119).
Roces, C.; Tourón, J.; González Torres, M.; Núñez Pérez, J.; González-Pineda, J.; González-Pumaraga, S.; y otros. (1998). Adaptación de la escala motivacional del MSLQ. V Congreso de Evaluación Psicológica. Málaga.

Sosa Sánchez-Cortés, S.; García Manso, A.; Sánchez Allende, J.; Moreno Díaz, P.; Reinoso Peinado, A. (2005). B-Learning y Teoría del Aprendizaje Constructivista en las Disciplinas Informáticas: Un esquema de ejemplo a aplicar. 3rd International Conference on Multimedia and Information and Communication TechnologiesinEducation, m-ICTE2005. Sevilla, España.

Suárez,R.J.M.;Anaya,N.D.(2004).Educación a distancia y presencial: diferencias en los componentes cognitivo y motivacional de estudiantes universitarios. RIED. Revista Iberoamericana de Educación a Distancia , 7, (65-75).

Torres, C. J.; Eberle, J. (2010). Student demographics and success in online learning environments. Emporia State Research Studies, 46 (1), (4-10).

Torres, N. L. C. (2006). La educación a distancia en México: ¿quién y cómo la hace? Apertura. Revista de innovación electrónica, 6 (4).

Tünnermann, B. C. (1999). La universidad ante los retos del siglo XXI. Educación superior y sociedad, 10 (1).

Tünnermann, B. C. (2003). La Universidad ante los retos del siglo XXI. México: Universidad Autónoma de Yucatán.

Tünnermann, B. C. (2007). Prólogo a Notas para un estudio comparado de la educación superior. En: López Segrera, F. Escenarios mundiales de la educación superior. Análisis global y estudios de casos. Consejo Latinoamericno de Ciencias Sociales.

Tünnermann, B. C. (2010). La educación permanente y su impacto en la educación superior. IISUE-UNAM/Universia, Ed. 
Revista Iberoamericana de Educación Superior (RIES), 1 (1), (120-133).

UNESCO (1998). Declaración Mundial Sobre la Educación Superior en El Siglo XXI: Visión y Acción. [en línea] Disponible en: www.unesco.org/education/educprog/ wche/declaration spa.htm (consulta 2007, 14 de enero).

UNISON (1999). La educación superior en el Siglo XXI: Visión y acción. Universidad de Sonora. [en línea] Disponible en: http://educadis.uson.mx/Educbibliovirt-doc/Articulos\%20Educacion/ Art-Educacion-varios/ES-siglo-XXITunnermann-Peon-Anaya-99.doc (consulta 2004, 10 de julio).

Vásquez, M. C. R. (2003). Deserción en Educación a distancia en Educación Superior. Miami: Virtual Educa 2003.

Vásquez, M. C. R.; Rodríguez, P. M. C. (2007). La deserción estudiantil en educación superior a distancia: perspectiva teórica y factores de incidencia. Revista Latinoamericana de Estudios Educativos, XXXVII (3-4), (107-122).
Vega, G. R. (2005). La educación continua a distancia en México: transformaciones y retos. Revista de la Educación Superior, XXXIV (1), (133).

Weinstein, C. E.; Mayer, R. E. (1986). The Teaching of learning strategies. En: Wittrock, M. Handbook of research on teaching. New York: MacMillan (315 327).

Yukselturk, E.; Bulut, S. (2009). Gender Differences in Self-Regulated Online Learning Environment. Educational Technology \& Society , 13 (3), (12-22).

Zubieta, G. J.; Cervantes, P. F.; Rojas, S. C. (2009). La deserción y el rezago en la Educación Superior a Distancia: signos promisorios en una Universidad Pública Mexicana. Observatorio de la Educación Virtual en América Latina y el Caribe. [en línea] Disponible en: www. e-continua.com/documentos/desercion educdistancia Mex UNAM Zub Cerv Rojas 2009.pdf (consulta 2010, 21 de enero). 


\section{PERFIL ACADÉMICO Y PROFESIONAL DE AUTOR}

Néstor Fernández Sánchez. Psicólogo educativo, egresado de la Universidad Nacional Autónoma de México con los grados de licenciatura y maestría. Académico dela misma Universidad desde 1976; formador de formadores, desarrollador, ejecutor y asesor de diversos cursos en Educación Abierta y a Distancia. Secretario Técnico de la Academia Mexicana de Profesionales de Educación Abierta y a Distancia A. C. y Presidente del Instituto de Educación Continua y Capacitación A. C.

E-mail: $\underline{\text { nfs@unam.mx }}$

\section{DIRECCIÓN DEL AUTOR}

Periférico Sur 7666 - 344, Rinconada Coapa.

Tlalpan, Distrito Federal, México. C. P. 14330.

Fecha de recepción del artículo: 23/03/11

Fecha de aceptación del artículo: 04/05/11

\section{Como citar este artículo:}

Fernández Sánchez, N. (2011). Promoción del cambio de estilos de aprendizaje y motivaciones en estudiantes de educación superior mediante actividades de trabajo colaborativo en Blended Learning. RIED. Revista Iberoamericana de Educación a Distancia, volumen 14, $\mathrm{n}^{\mathrm{O}}$ 2, pp. 189-208. 